\title{
Cerebrospinal fluid $A \beta 42$, t-tau, and $p$-tau levels in the differential diagnosis of idiopathic normal-pressure hydrocephalus: a systematic review and meta-analysis
}

\author{
Zhongyun Chen ${ }^{1}$, Chunyan Liu', Jie Zhang ${ }^{1}$, Norman Relkin², Yan Xing ${ }^{1 *}$ and Yanfeng Li $i^{*}$
}

\begin{abstract}
Objectives: The purpose of this systematic review and meta-analysis was to evaluate the performance of cerebrospinal fluid (CSF) beta amyloid 42 (Aß42), total tau (t-tau), and phosphorylated tau (p-tau) as potential diagnostic biomarkers for idiopathic normal-pressure hydrocephalus (iNPH) and to assess their utility indistinguishing patients with iNPH from those with Alzheimer disease (AD) and healthy normal controls.

Methods: Studies were identified by searching PubMed, Embase, the Cochrane Library, Web of Science, Chinese National Knowledge Infrastructure (CNKI), Wanfang Chinese Periodical Database, VIP Chinese database, and Chinese Bio-medicine Database (CBM) before August 2016. The standardized mean difference (SMD) and 95\% confidence interval (Cl), comparing CSF A 42 , t-tau, and p-tau levels between iNPH, AD and healthy controls, were calculated using random-effects models. Subgroup analyses were created according to ethnicity (Caucasian or Asian) and CSF type (lumbar or ventricular), and the publication bias was estimated using Egger's test and the Begg's test.

Results: A total of 10 studies including 413 patients with iNPH, 186 patients with AD and 147 healthy controls were included in this systematic review and meta-analysis. The concentrations of CSF t-tau, and p-tau were significantly lower in iNPH patients compared to $\mathrm{AD}(\mathrm{SMD}=-1.26,95 \% \mathrm{Cl}-1.95$ to $-0.57, \mathrm{P}=0.0004 ; \mathrm{SMD}=-1.54,95 \% \mathrm{Cl}$ -2.34 to $-0.74, P=0.0002$, respectively) and lower than healthy controls (SMD $=-0.80,95 \% \mathrm{Cl}-1.50$ to -0.09 , $P=0.03 ; \mathrm{SMD}=-1.12,95 \% \mathrm{Cl}-1.38$ to $-0.86, P<0.00001$, respectively). Patients with iNPH had significantly lower A 342 levels compared with controls (SMD $=-1.14,95 \% \mathrm{Cl}-1.74$ to $-0.55, P=0.0002)$, and slightly higher $\mathrm{A} \beta 42$ levels compared with $A D$ patients $(S M D=0.32,95 \% \mathrm{Cl} 0.00-0.63, P=0.05)$. Subgroup analyses showed that the outcomes may have been influenced by ethnicity and CSF source. Compared to AD, overall sensitivity in differentiating iNPH was 0.813 (95\% Cl 0.636-0.928) for A $342,0.828$ (95\% Cl 0.732-0.900) for t-tau, 0.943 (95\% Cl 0.871-0.981) for $\mathrm{p}$-tau. Relative to AD, overall specificity in differentiating iNPH was $0.506(95 \% \mathrm{Cl} 0.393-0.619)$ for $\mathrm{A} \beta 42,0.842(95 \% \mathrm{Cl}$ 0.756-0.907) for t-tau, 0.851 (95\% Cl 0.767-0.914) for p-tau.
\end{abstract}

Conclusion: The results of our meta-analysis suggest that iNPH may be associated with significantly reduced levels of CSF A 342 , t-tau and p-tau compared to the healthy normal state. Compared to AD, both t-tau and p-tau were significantly decreased in iNPH, but CSF Aß42 was slightly increased. Prospective studies are needed to further assess

\footnotetext{
*Correspondence: drxingyan@163.com; doctorliyf@163.com

${ }^{1}$ Department of Neurology, Aviation General Hospital of China Medical University \& Beijing Institute of Translational Medicine, Chinese Academy of Sciences, No. 3 Anwai Beiyuan Road, Chaoyang District, Beijing 100012, China

${ }^{3}$ Department of Neurology, Peking Union Medical College Hospital,

Beijing, China

Full list of author information is available at the end of the article
} 
the clinical utility of these and other CSF biomarkers in assisting in the diagnosis of iNPH and differentiating it from AD and other neurodegenerative disorders.

Keywords: CSF biomarkers, Idiopathic normal-pressure hydrocephalus, Alzheimer's disease, Meta-analysis, Systematic review

\section{Background}

Normal pressure hydrocephalus (NPH) was first described in 1965 by Hakim, Adams and colleagues [1] as a syndrome of cerebral ventricular enlargement occurring in adults without elevated cerebrospinal fluid (CSF) pressure or macroscopic obstruction to CSF flow. Early studies identified NPH as a progressive but treatable disorder that often presents with the classical symptom triad of gait disturbance, dementia, and urinary incontinence. This condition is considered idiopathic NPH (iNPH) when there is no identifiable antecedent cause and secondary NPH $(\mathrm{sNPH})$ when events such as severe head trauma, subarachnoid hemorrhage or meningitis precede its onset.

Recent studies have reported prevalence rates of iNPH ranging from 0.51 to $5.9 \%$ in the elderly population that increase with advancing age. This suggests that iNPH is much more common than previously recognized [2-4]. It is extremely underdiagnosed throughout most of the world [5] and less than $10-20 \%$ of patients with iNPH get appropriate specialized treatment $[6,7]$. This is particularly unfortunate because iNPH and sNPH can be effectively treated by neurosurgical placement of a shunt, which leads to improvement or stabilization of symptoms in upwards of $80 \%$ of accurately diagnosed patients [8].

An important factor in the under-diagnosis of iNPH is that early clinical features may be subtle and its manifestations can overlap those of other neurological disorders such as $\mathrm{AD}$ and normal brain aging. Therefore, finding sensitive and specific tools for early and accurate differential diagnosis is vital for improving the detection and care of patients with iNPH.

Analysis of CSF biomarkers is performed as part of many neurodegenerative research studies and is increasingly employed in the clinical diagnostic work-up when neurodegenerative disorders are suspected. $A \beta 42, t$-tau, and $\mathrm{p}$-tau have been widely validated as CSF biomarkers for AD diagnosis. In particular, a pattern of reduced CSF A $\beta 42$ with elevated CSF $p$-tau and $t$-tau is strongly associated with $\mathrm{AD}$ [9]. The $\mathrm{A} \beta 42$ protein is closely linked to AD pathology as the central component of extracellular neuritic plaques [10]. Tau is an intracellular microtubuleassociated protein and its total level in CSF is thought to reflect the extent of ongoing neuronal death. Hyperphosphorylated forms of tau (p-tau) are more closely associated with neurofibrillary tangle formation in $\mathrm{AD}$ and measurement of CSF p-tau therefore adds specificity for $\mathrm{AD}$ to the CSF biomarker profile $[10,11]$.

Comparably fewer CSF biomarker studies have been performed on iNPH patients and there has been only moderate consistency among those performed. Some studies have reported that patients with iNPH have low A $\beta 42$ similar to those in AD, but without increased $t$-tau and p-tau levels. There has been speculation that this pattern might be useful for differentiating iNPH from $\mathrm{AD}[12,13]$. However, other studies did not reach the same conclusion [14, 15]. Considering these inconsistent results, we conducted a meta-analysis to evaluate whether CSF A $\beta 42$, $t$-tau, and $p$-tau levels are of value in the differential diagnosis of iNPH from AD and healthy normal controls.

\section{Methods \\ Literature search}

We did asystematic review and meta-analysis according to the PRISMA guidelines [16]. Two authors searched PubMed, Embase, the Cochrane Library, Web of Science, Chinese National Knowledge Infrastructure (CNKI), Wanfang Chinese Periodical Database, VIP Chinese database, and Chinese Bio-medicine Database (CBM) for relevant articles published before August 2016 by using Medical Subject Heading (MeSH) terms and the following free text terms: "(normal pressure OR normotensive) AND hydrocephal") OR Hydrocephalus, Normal Pressure [Mesh]" AND "((biological) AND markers) OR biomarker OR CSF OR cerebrospinal" AND "A $\beta 42$ OR Abeta42 OR Abeta-42 OR A $11-42$ OR t-tau OR p-tau OR tau OR phospho-tau OR phosphorylated tau".

The search was confined to human studies published in English and Chinese. The titles and abstracts of each article were scanned independently by two authors (ZYC and $\mathrm{CYL}$ ) to exclude studies that were clearly irrelevant. The full text of the remaining studies were then retrieved and assessed for eligibility according to the inclusion criteria. Any disagreement was resolved by discussion with a third author (JZ).

\section{Study selection}

Studies were eligible for the analysis if they fulfill the following criteria: (1) case-control studies which compared the CSF levels of A $\beta 42$ and/or $\mathrm{t}$-tau and/or p-tau between iNPH patients and AD patients or healthy controls; 
(2) clearly stated $\mathrm{iNPH}$ and AD diagnostic criteria (see Additional file 1: Table S1); (3) original articles containing independent data, and data were expressed as mean and standard deviation (SD) or median and interquartile range (IQR).

The exclusion criteria for the study were as follows: (1) abstracts, reviews, case reports, animal experiments, experts' opinions and commentaries; (2) duplicate publications of the same dataset; (3) papers not specifically focused on iNPH (i.e.: NPH or sNPH).

\section{Data extraction}

Two authors extracted data from the included articles, which included the following: the first author's name, year of publication, country, number of cases and controls, age (mean $\pm \mathrm{SD}$ or median and interquartile range), the number of females and males, the CSF levels of $A \beta 42$, $t$-tau and p-tau concentration (mean $\pm S D$ or median and interquartile range), analytical technology, and CSF source.

\section{Quality evaluation}

The Newcastle-Ottawa Quality Assessment Scale (NOS) was used to assess the quality of each included study, and was performed by two authors independently, with a third author consulted in case of discrepancy. Three major components were collected: (1) the selection (0-4 points); (2) the exposure (0-3 points); 3 ) the comparability ( $0-2$ points). Higher scores represent better quality in methodology. All studies in this systematic review had scores greater than or equal to seven, indicating good qualities.

\section{Statistical analysis}

Statistical analyses were performed using Review Manager 5.1.2 (Cochrane Collaboration, Oxford, UK), Meta DiSc 1.4 version (Cochrane Collaboration, Oxford, UK) and Stata 12.0 (Stata Corp, College Station, Texas, USA). Data given as median and IQR were converted into mean \pm SD in accordance with the protocol provided by Wan et al. [17]. In studies where patients with iNPH were divided into shunt responder and shunt non-responders, we combined the two datasets for the purpose of the current evaluation [18].

The standardized mean difference (SMD) and the corresponding $95 \% \mathrm{CI}$ were used as the main effect measure. Heterogeneity across the studies was estimated by using the Chi square based Cochran $\mathrm{Q}$ and the $I^{2}$ test statistics [19]. The heterogeneity was considered statistically significant if $P<0.1$ or $I^{2}>50 \%$, a random-effects model (DerSimonian-Laird method) of analysis was used; otherwise, the fixed-effects model (Mantel-Haenszel method) was applied instead. Sub-groups were created according to ethnicity (Caucasian or Asian) and CSF source (lumbar or ventricular). Sensitivity analysis was performed by removing studies one by one to detect its influence on pooled ORs. The Egger's test and the Begg's test were used to estimate the severity of publication bias [20]. Where publication bias existed, we used the Trim and Fill method to correct it. The overall sensitivity, specificity, positive likelihood ratio (PLR), and negative likelihood ratio (NLR), as well as their corresponding 95\% CIs, were pooled based on the random effects model. In addition, the area under the curve (AUC) and Q* index were calculated to evaluate the diagnostic test accuracy. All statistical tests were 2 -sided, and statistical significance was defined as $P<0.05$.

\section{Results}

\section{Included studies}

A total of 121 relevant articles were identified in the initial search. Seventy-four articles remained after removal of duplicate studies and 28 articles were excluded based on titles and abstracts. After systematically reviewing the remaining 46 full-text articles, 36 articles were excluded for not fulfilling our inclusion criteria. Finally, ten articles met stringent search criteria for data analysis and three articles were included in the diagnostic analysis. A detailed flow chart of the search and selection process is depicted in Fig. 1.

\section{Study characteristics}

The characteristics of the ten studies included in the meta-analysis are listed in Table 1. A total of 413 patients with iNPH, 186 patients with AD, and 147 healthy controls were included in this meta-analysis. 2 studies were performed in the United States [21, 22], 2 in Japan [23, 24], 2 in Sweden [12, 25], 1 in Greece [13], 1 in Finland [26], 1 in South Korea [27] and 1 in Italy [28]. These studies were published between 2007 and 2015. With respect to the assay method used to measure CSF A $\beta 42$, $\mathrm{t}$-tau, $\mathrm{p}$-tau levels, 9 studies were performed using ELISA methodology and 1 was conducted using other methods.

\section{Meta-analysis \\ Pooled analysis (Table 2)}

A 342 in iNPH versus AD/healthy controls Seven studies, including 268 patients with iNPH and 186 patients with AD and 6 studies, including 193 patients with iNPH and 147 healthy controls were used in the meta-analysis, respectively. A random-effect model was used to calculate pooled SMD because of highly significant heterogeneity among those studies (iNPH versus $\mathrm{AD}, P<0.06, I^{2}=50 \%$; iNPH versus healthy controls, $P=0.0003, I^{2}=79 \%$ ). Patients with iNPH showed significantly decreased A $\beta 42$ levels compared with healthy controls $(\mathrm{SMD}=-1.14$, 


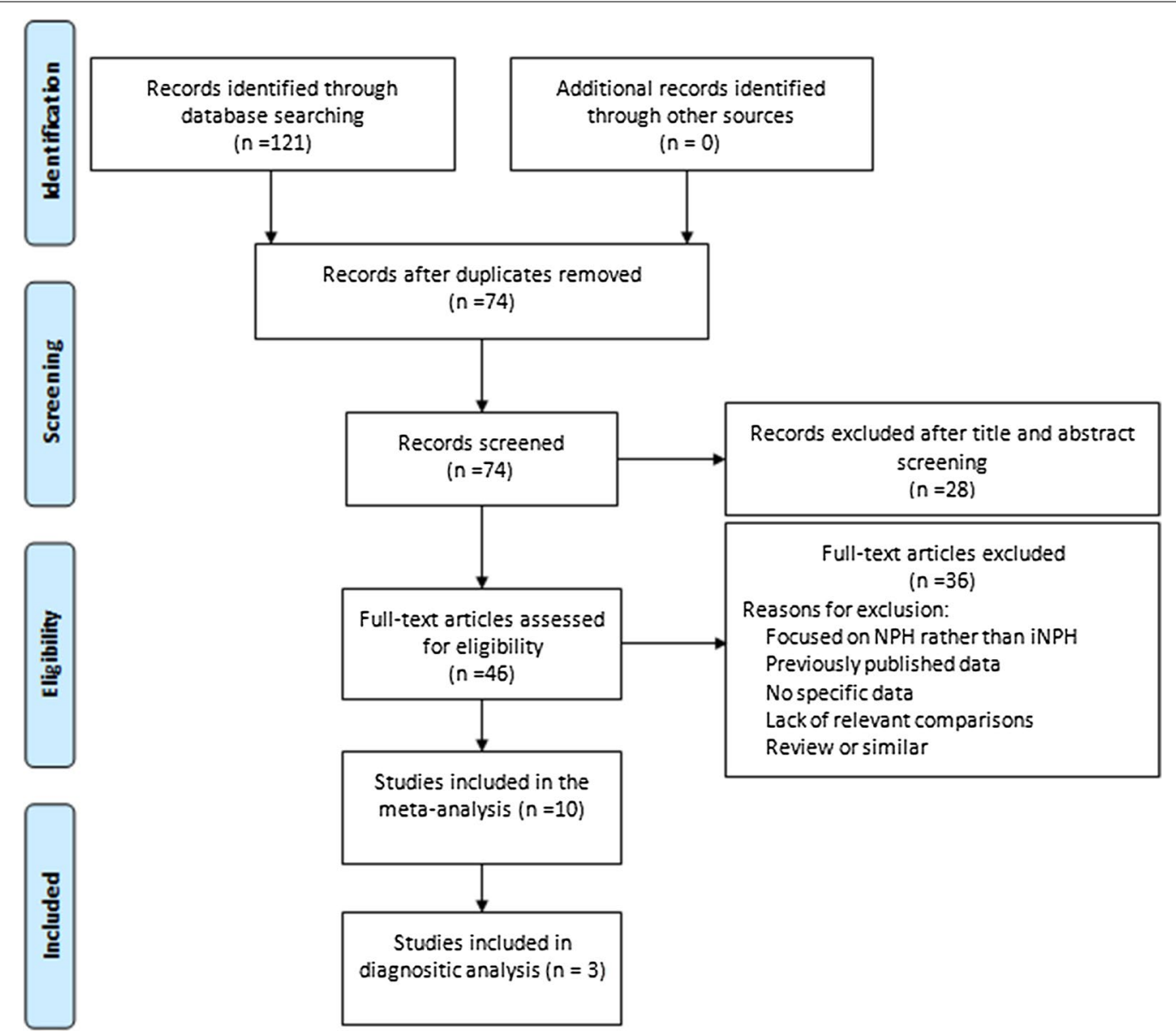

Fig. 1 Flow chart of the search and selection process

$95 \% \mathrm{CI}-1.74$ to $-0.55, P=0.0002$ ), and slightly increased $\mathrm{A} \beta 42$ levels compared with $\mathrm{AD}$ patients $(\mathrm{SMD}=0.32$, 95\% CI 0.00-0.63, $P=0.05$ ).

T-tau in iNPH versus AD/healthy controls Eight studies reported values for CSF t-tau in $323 \mathrm{iNPH}$ patients and $206 \mathrm{AD}$ patients and 6 studies reported values for CSFttau in 193 iNPH patients and 147 healthy controls. A significant heterogeneity across studies was found (iNPH versus $\mathrm{AD}, P<0.00001, I^{2}=90 \%$; iNPH versus healthy controls, $P<0.00001, I^{2}=86 \%$ ), thus the random-effects model was used to calculate the pooled SMD. T-tau levels were significantly lower in iNPH patients than in AD $(\mathrm{SMD}=-1.26,95 \% \mathrm{CI}-1.95$ to $-0.57, P=0.0004)$ and significantly lower than in healthy controls $(\mathrm{SMD}=-0.80$, $95 \% \mathrm{CI}-1.50$ to $-0.09, P=0.03)$.

P-tau in iNPH versus AD/healthy controls Mean P-tau values of iNPH patients were compared with $A D$ patients in 8 articles, including $323 \mathrm{iNPH}$ patients and $206 \mathrm{AD}$ patients. 6 studies reported CSF values for p-tau in 194
iNPH patients and 147 healthy controls. A significant heterogeneity across studies was found in iNPH versus AD $\left(P<0.00001, I^{2}=92 \%\right)$, while no heterogeneity was found between iNPH and healthy controls $\left(P=0.64, I^{2}=0 \%\right)$. P-tau levels were significantly lower in iNPH patients than in $\mathrm{AD}$ (SMD $=-1.54,95 \% \mathrm{CI}-2.34$ to -0.74 , $P=0.0002)$ and significantly lower than in healthy controls $(\mathrm{SMD}=-1.12,95 \% \mathrm{CI}-1.38$ to $-0.86, P<0.00001)$.

\section{Subgroup analysis}

A subgroup analysis was performed according to the categories of country (Asia or others) and CSF source (lumbar or ventricular) (see Table 2).

Of the ten studies included in this meta-analysis, 3 studies were performed in Asian populations, whereas 7 studies were performed in Caucasian groups. For $A \beta 42$ levels in iNPH versus AD, the pooled SMD was 0.75 (95\% CI $0.27-1.22, P=0.002)$ in Asian groups and 0.17 (95\% CI -0.07 to $0.40, P=0.16$ ) in Caucasian groups. For A 342 levels in $\mathrm{iNPH}$ versus healthy controls, the pooled SMD was -0.17 (95\% CI -0.96 to $0.63, P=0.58$ ) in Asian 


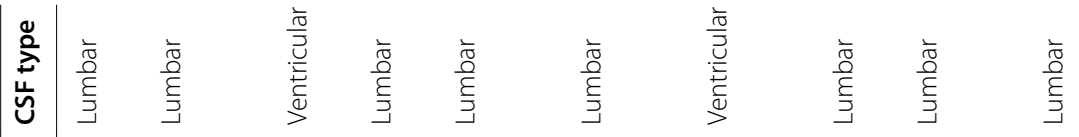

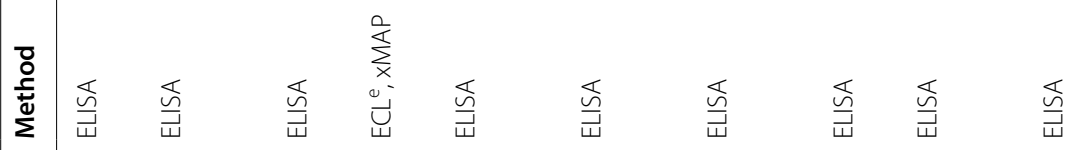

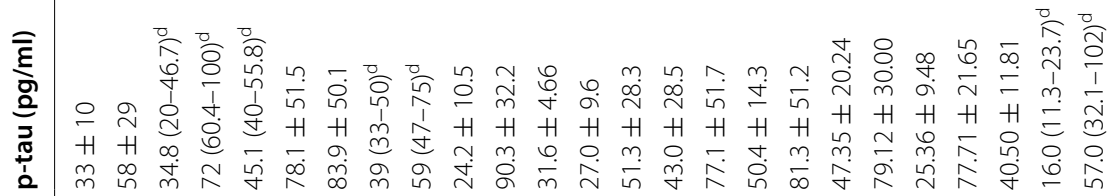

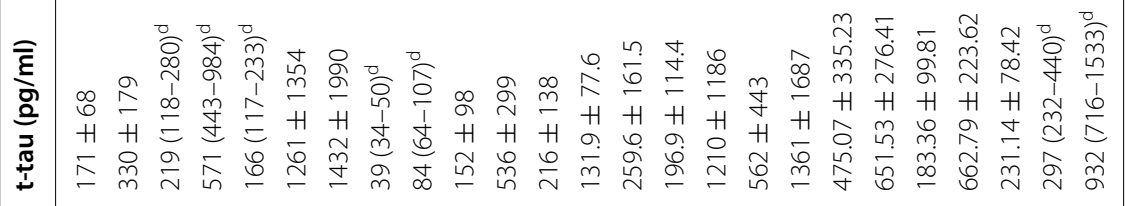

产

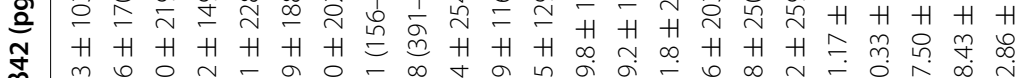

妾

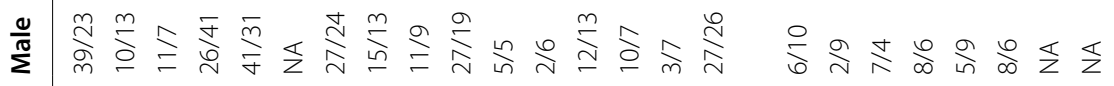

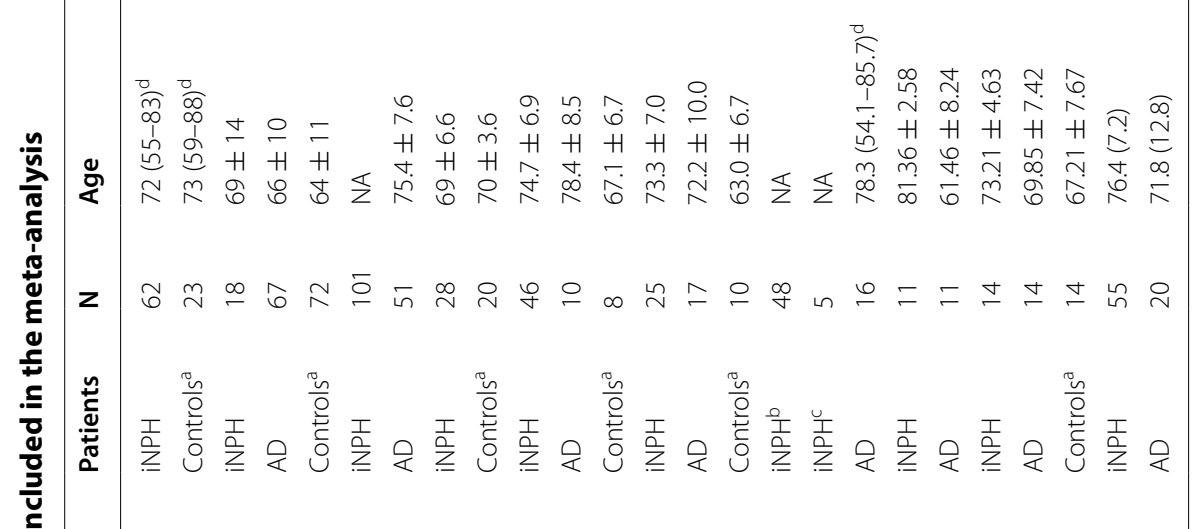

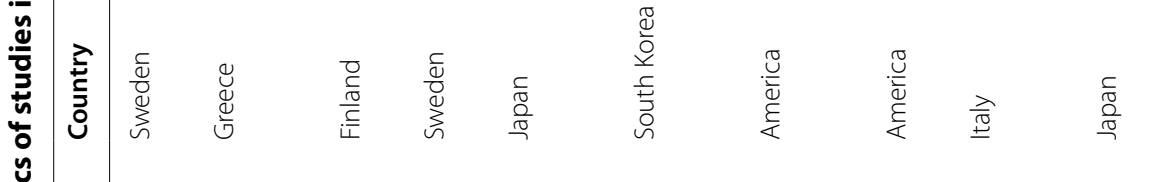

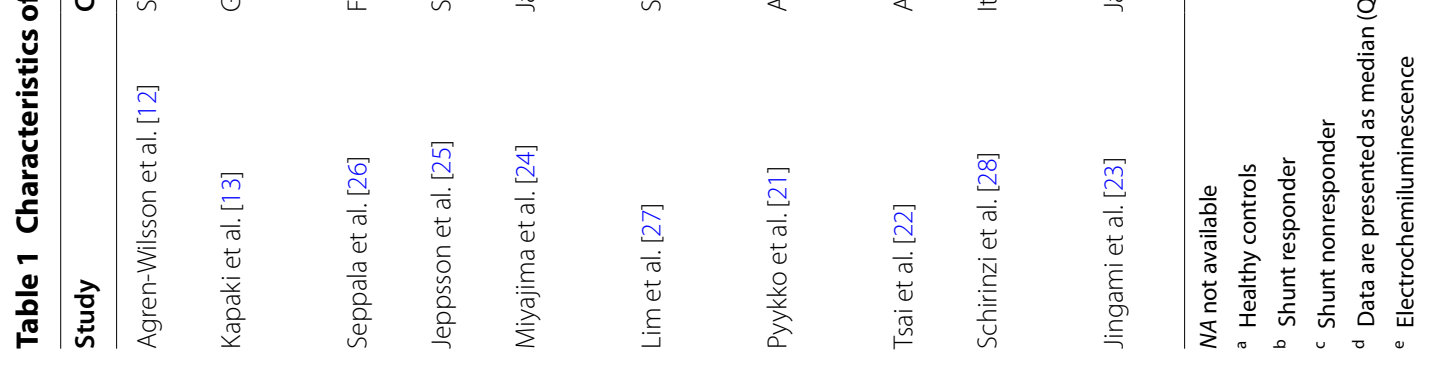




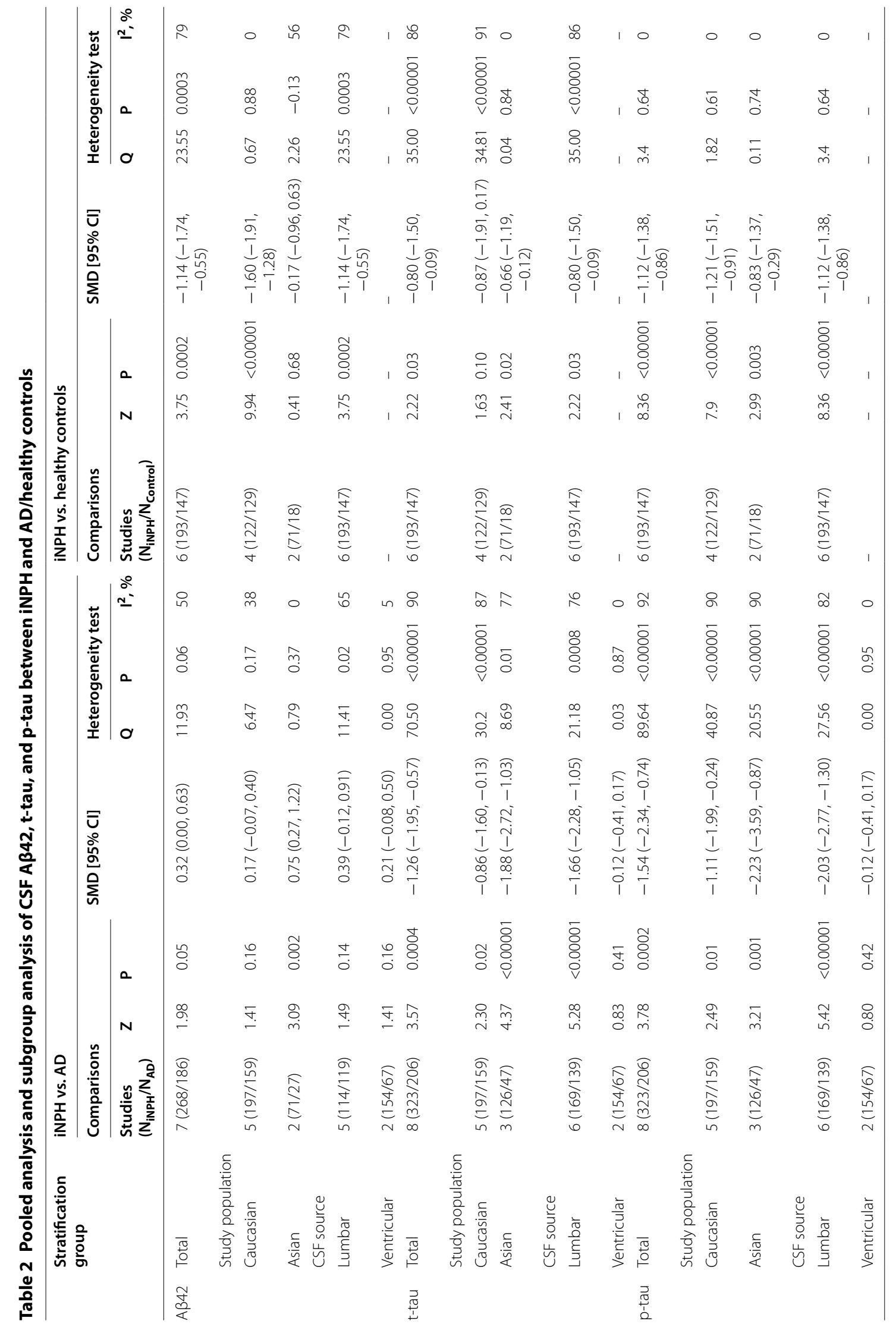


groups and -1.60 (95\% CI -1.91 to $-1.28, P<0.00001)$ in Caucasian groups. For $\mathrm{t}$-tau levels in iNPH versus AD, the pooled SMD was -1.88 (95\% CI -2.72 to -1.03 , $P<0.00001)$ in Asian groups and -0.86 (95\% CI -1.60 to $-0.13, P=0.02)$ in Caucasian groups. For $\mathrm{t}$-tau levels in iNPH versus healthy controls, the pooled SMD was $-0.66(95 \% \mathrm{CI}-1.19$ to $-0.12, P=0.02)$ in Asian groups and -0.87 (95\% CI -1.91 to $0.17, P=0.10)$ in Caucasian groups. For p-tau levels in iNPH versus AD, the pooled SMD was -2.23 (95\% CI -3.59 to $-0.87, P=0.001$ ) in Asian groups and -1.11 (95\% CI -1.99 to -0.24 , $P=0.01$ ) in Caucasian groups. For p-tau levels in iNPH versus healthy controls, the pooled SMD was -0.83 $(95 \% \mathrm{CI}-1.37$ to $-0.29, P=0.003)$ in Asian groups and $-1.21(95 \% \mathrm{CI}-1.51$ to $-0.91, P<0.00001)$ in Caucasian groups.

Of the ten studies included in this meta-analysis, 8 studies were performed using lumbar CSF while 2 studies were using ventricular CSF. For $\mathrm{A} \beta 42$ levels in $\mathrm{iNPH}$ versus $\mathrm{AD}$, the pooled SMD was $0.39(95 \% \mathrm{CI}-0.12$ to 0.91 , $P=0.14)$ in lumbar CSF groups and $0.21(95 \% \mathrm{CI}-0.08$ to $0.50, P=0.16)$ in ventricular CSF groups. For $\mathrm{t}$-tau levels in $\mathrm{iNPH}$ versus $\mathrm{AD}$, the pooled SMD was -1.66
(95\% CI -2.28 to $-1.05, P<0.00001)$ in lumbar CSF groups and $-0.12(95 \% \mathrm{CI}-0.41$ to $0.17, P=0.41)$ in ventricular CSF groups. For p-tau levels in iNPH versus $\mathrm{AD}$, the pooled SMD was -2.03 (95\% CI -2.77 to -1.30 , $P<0.00001)$ in lumbar CSF groups and -0.12 (95\% CI -0.41 to $0.17, P=0.42)$ in ventricular CSF groups.

\section{Sensitivity analysis}

Sensitivity analysis was performed by removing studies one by one and comparing the pooled estimate from the remaining studies with the pooled estimate from all studies. Sensitivity analysis of A $\beta 42$ levels between iNPH and healthy controls, $t$-tau levels between $\mathrm{iNPH}$ and AD, and p-tau levels between iNPH and AD/healthy controls revealed that the direction and magnitude of pooled estimates did not change significantly, indicating that the results of the meta-analysis were relatively robust. In contrast, sensitivity analysis of A $\beta 42$ levels in iNPH and $\mathrm{AD}$, as well as t-tau levels in iNPH and healthy controls revealed that the pooled estimates were different when the leave-one-out approach was used. This suggests that results of those between-groups analyses were not stable and reliable (see Figs. 2, 3, 4, 5, 6, 7).

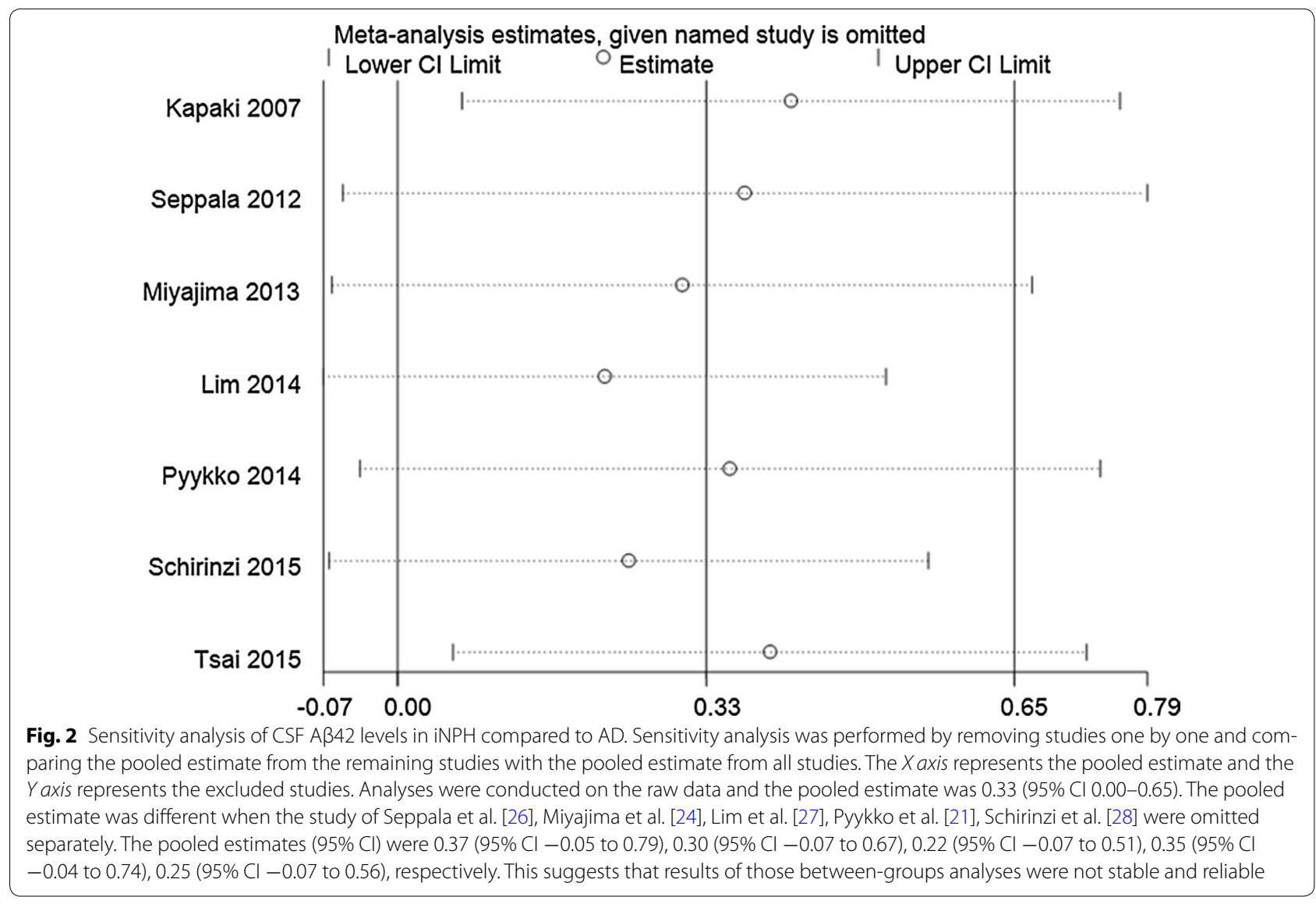




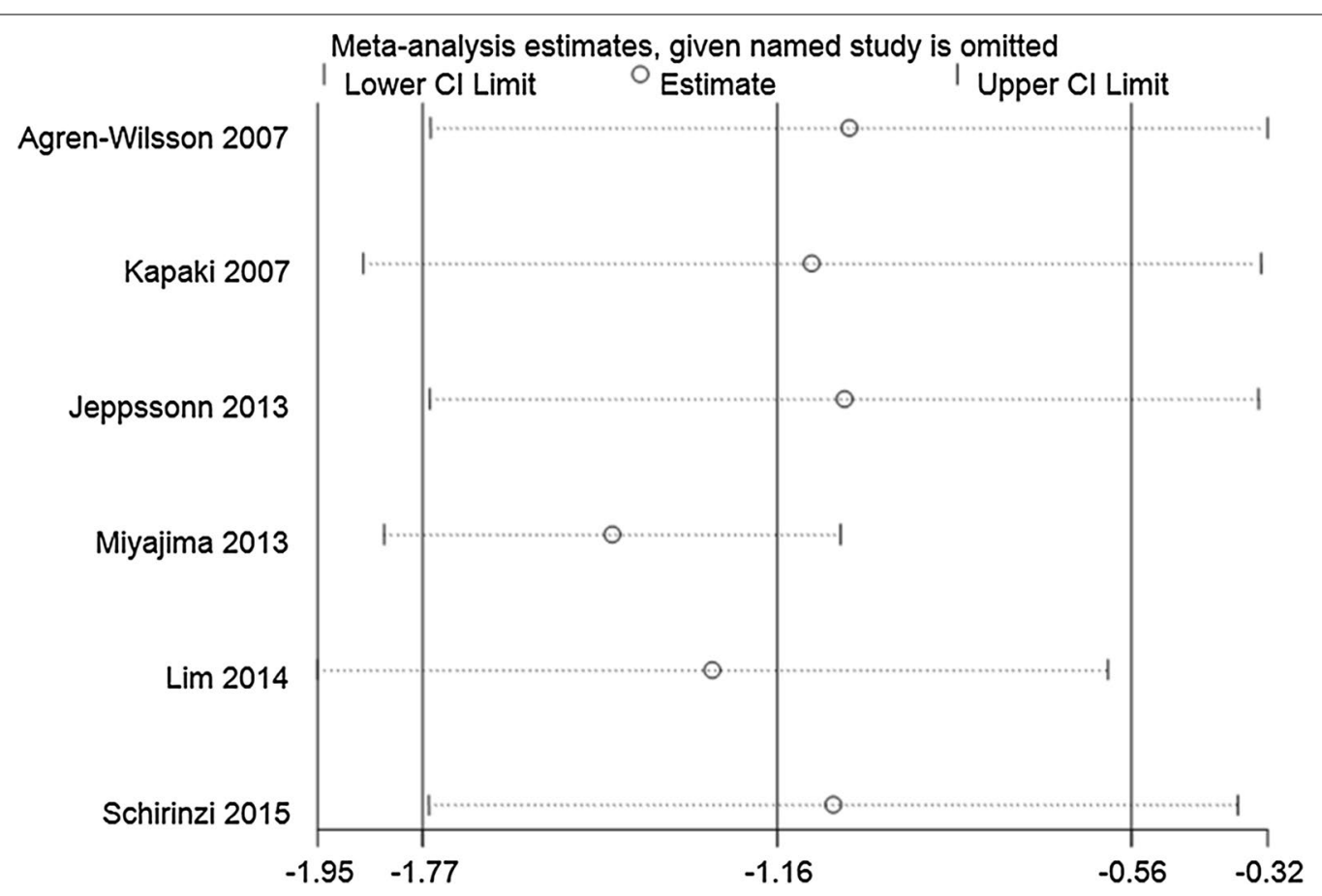

Fig. 3 Sensitivity analysis of CSF AB42 levels in iNPH compared to healthy controls. Analyses were conducted on the raw data and the pooled estimate was $-1.16(95 \% \mathrm{Cl}-1.77$ to -0.56$)$. The direction and magnitude of pooled estimates did not change significantly after removing studies one by one, indicating that the results of the meta-analysis were relatively robust

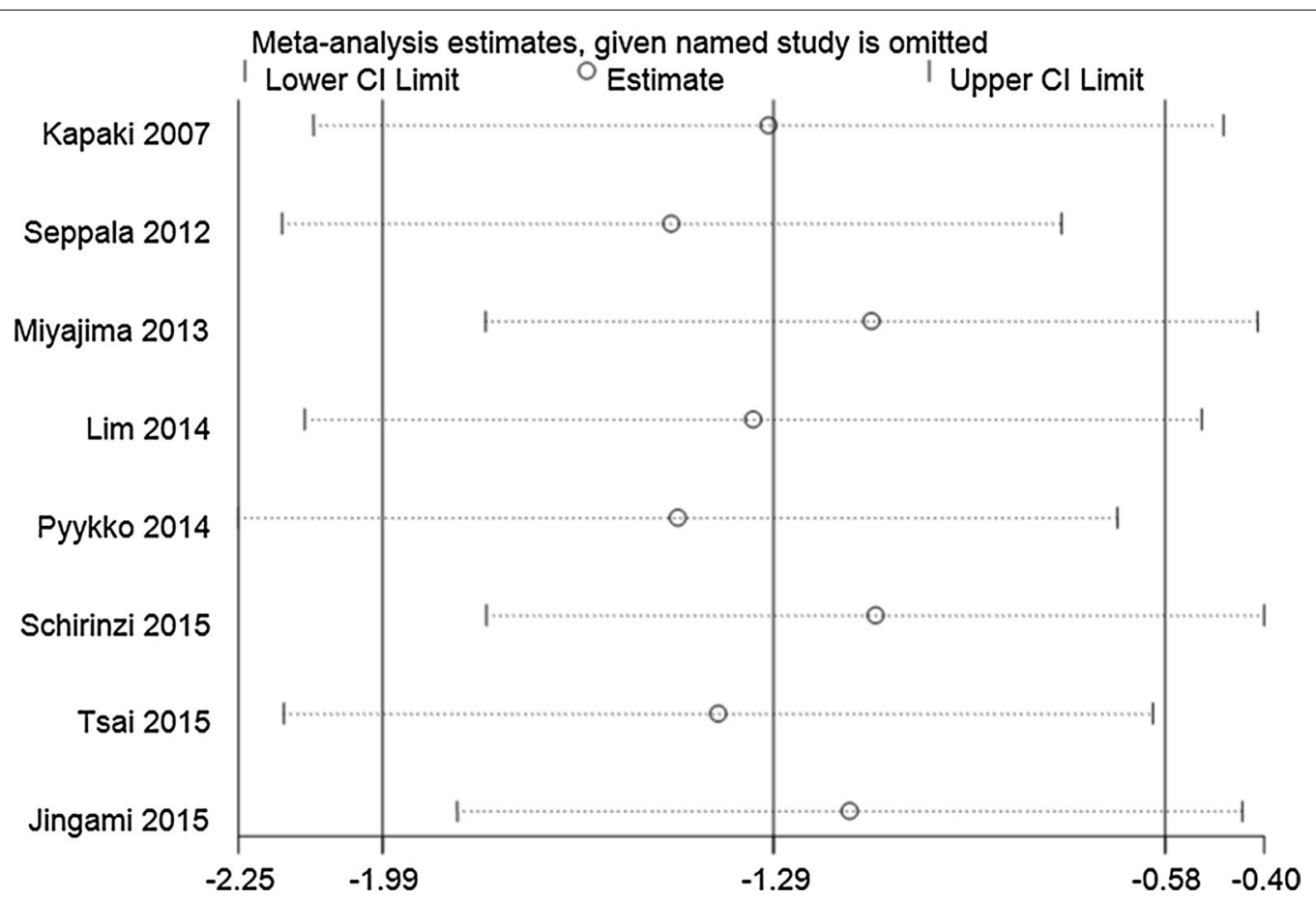

Fig. 4 Sensitivity analysis of CSF t-tau levels in iNPH compared to AD. Analyses were conducted on the raw data and the pooled estimate was $-1.29(95 \% \mathrm{Cl}-1.99$ to -0.58$)$. The direction and magnitude of pooled estimates did not change significantly after removing studies one by one, indicating that the results of the meta-analysis were relatively robust 


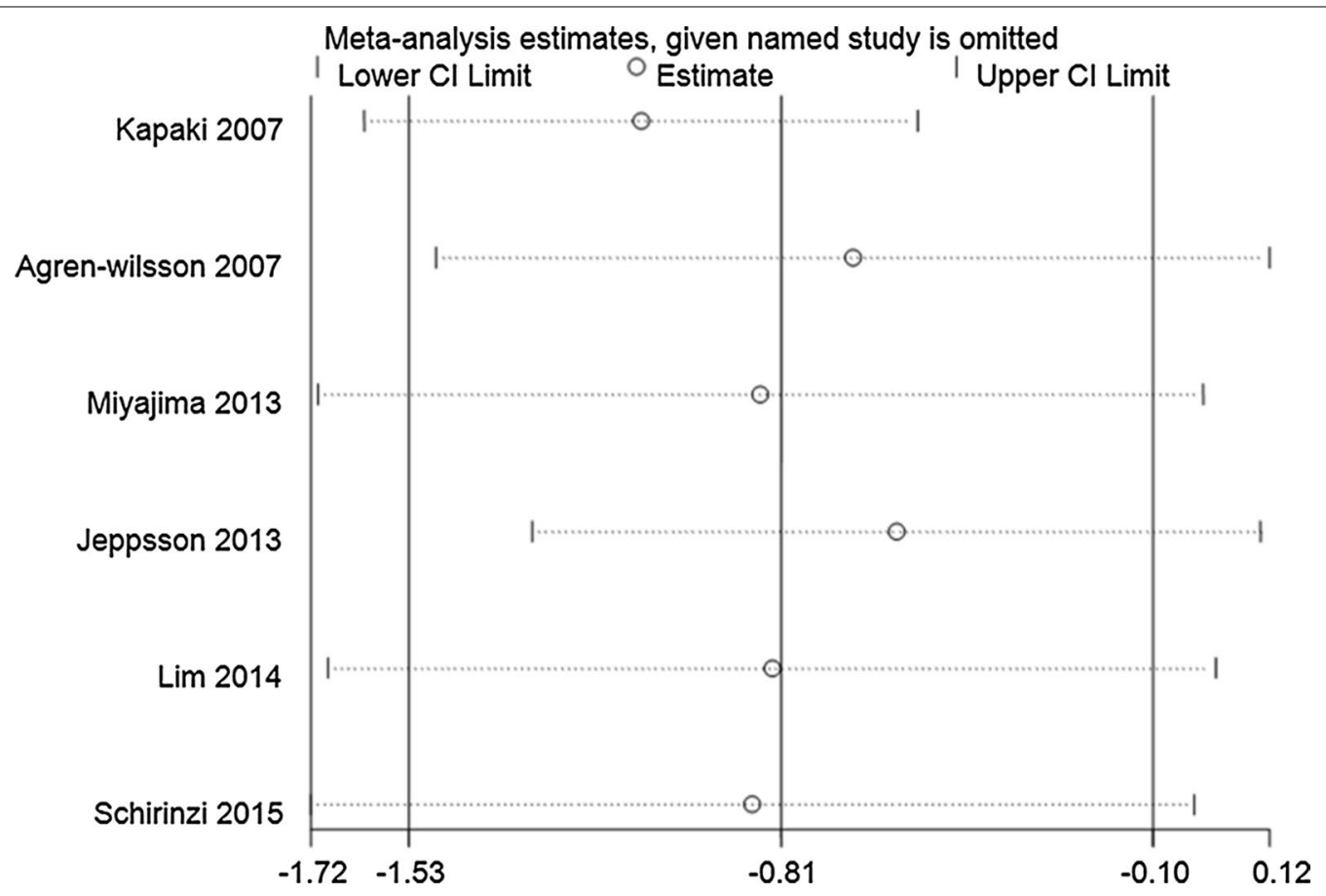

Fig. 5 Sensitivity analysis of CSF t-tau levels in iNPH compared to healthy controls. Analyses were conducted on the raw data and the pooled estimate was $-0.81(95 \% \mathrm{Cl}-1.53$ to -0.10$)$. The pooled estimate was different when the study of Agren-Wilsson et al. [12], Jeppsson et al. [25], Lim et al. [27] were omitted separately. The pooled estimates ( $95 \% \mathrm{Cl})$ were $-0.68(95 \% \mathrm{Cl}-1.47$ to 0.12$),-0.59(95 \% \mathrm{Cl}-1.29$ to 0.11$)$ and $-0.83(95 \%$ $\mathrm{Cl}-1.68$ to 0.21 ), respectively. This suggests that results of those between-groups analyses were not stable and reliable

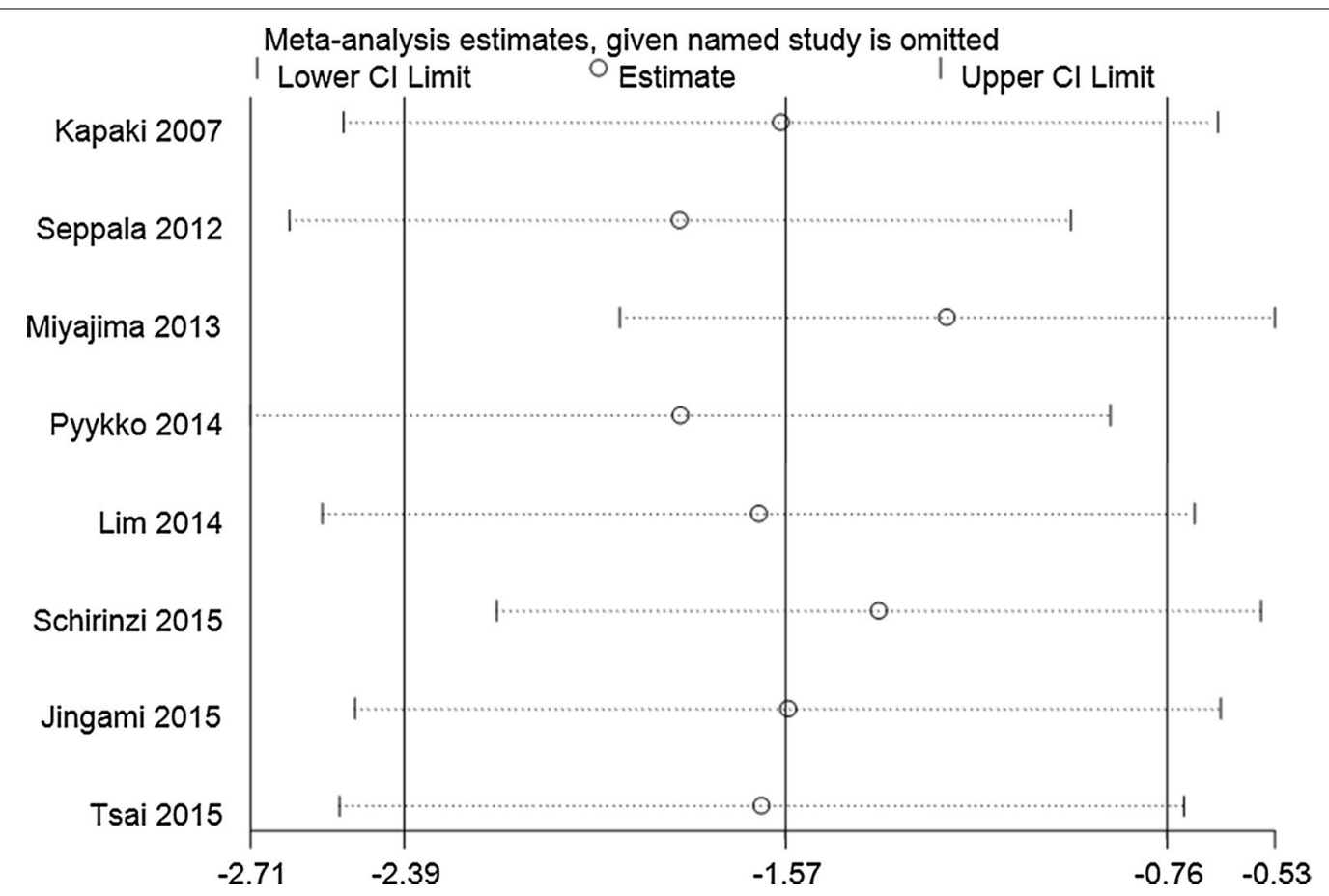

Fig. 6 Sensitivity analysis of CSF p-tau levels in iNPH compared to AD. Analyses were conducted on the raw data and the pooled estimate was $-1.57(95 \% \mathrm{Cl}-2.39$ to -0.76$)$. The direction and magnitude of pooled estimates did not change significantly after removing studies one by one, indicating that the results of the meta-analysis were relatively robust 


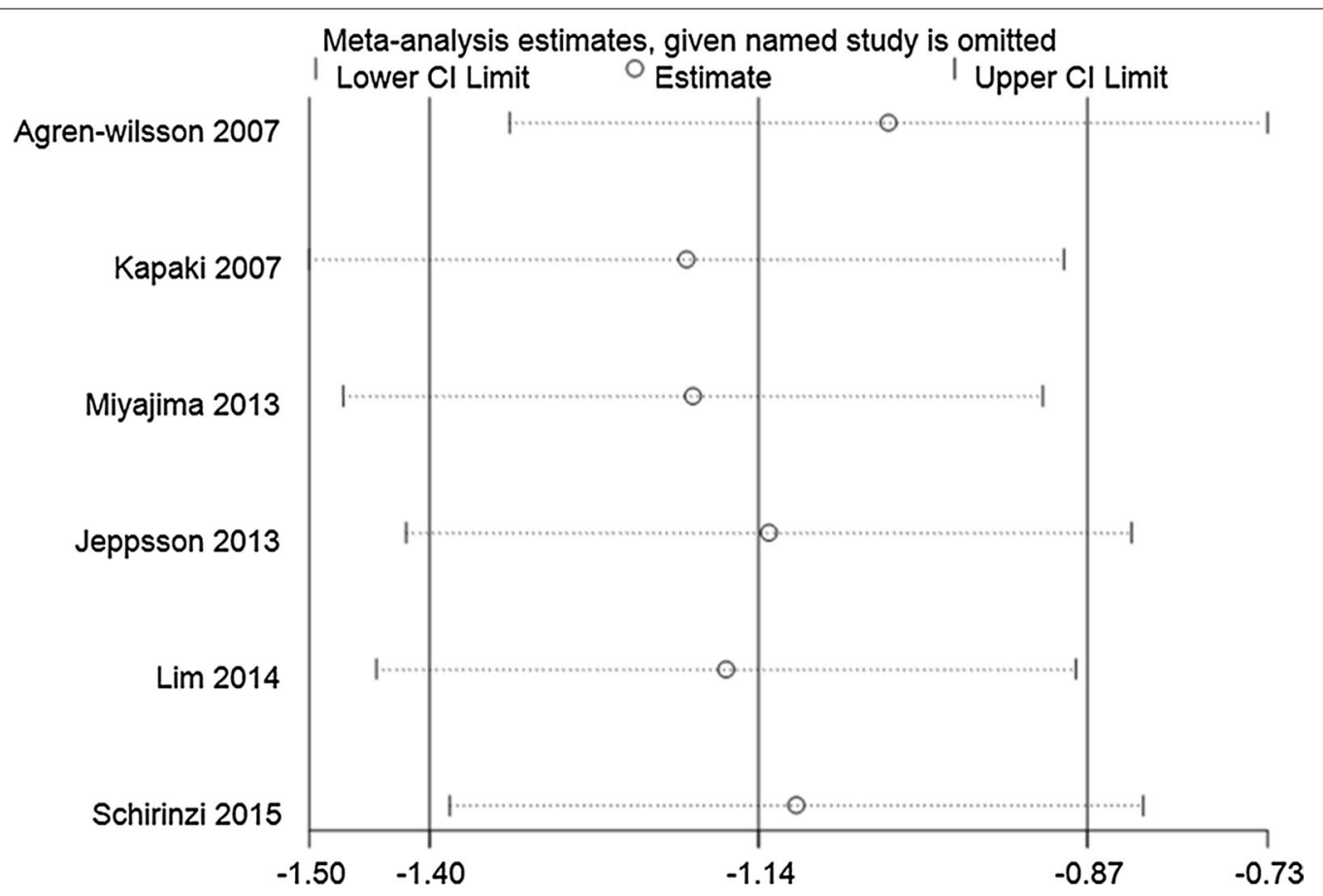

Fig. 7 Sensitivity analysis of CSF p-tau levels in iNPH compared to healthy controls. Analyses were conducted on the raw data and the pooled estimate was $-1.14(95 \% \mathrm{Cl}-1.40$ to -0.87$)$. The direction and magnitude of pooled estimates did not change significantly after removing studies one by one, indicating that the results of the meta-analysis were relatively robust

\section{Publication bias}

Begg and Egger tests were performed to assess for publication bias of the included studies and provide statistical evidence of publication funnel plot symmetry. Results showed that no significant publication bias was found in CSF A $\beta 42$ levels between iNPH and AD (Begg's test: $\mathrm{Z}=0.60, \mathrm{P}=0.548$; Egger's test: $\mathrm{t}=0.73, \mathrm{P}=0.500), \mathrm{A} \beta 42$ levels between iNPH and healthy controls (Begg's test: $\mathrm{Z}=1.13, \mathrm{P}=0.260$; Egger's test: $\mathrm{t}=0.96, \mathrm{P}=0.390$ ), $\mathrm{t}$-tau levels between iNPH and healthy controls (Begg's test: $\mathrm{Z}=0.00, \mathrm{P}=1.000$; Egger's test: $\mathrm{t}=-0.44, \mathrm{P}=0.686$ ), $\mathrm{p}$-tau levels between iNPH and healthy controls (Begg's test: $\mathrm{Z}=0.00, \mathrm{P}=1.000$; Egger's test: $\mathrm{t}=0.55, \mathrm{P}=0.609$ ). However evidence of publication bias was found in CSF $\mathrm{t}$-tau levels between iNPH and AD (Begg's test: $\mathrm{Z}=0.87$, $\mathrm{P}=0.386$; Egger's test: $\mathrm{t}=-2.77, \mathrm{P}=0.032)$ and $\mathrm{p}$-tau levels between $\mathrm{iNPH}$ and $\mathrm{AD}$ (Begg's test: $\mathrm{Z}=1.86$, $\mathrm{P}=0.063$; Egger's test: $\mathrm{t}=-3.69, \mathrm{P}=0.010$ ). We therefore used the Trim and Fill method to correct it. There was no significant change in the results after using the trim and fill method, which suggested that the influence of publication bias on stability of results was weak.

\section{Diagnostic results of included studies}

Detailed data regarding the sensitivity, specificity and other diagnostic results were presented in Table 3 .
Compared to $\mathrm{AD}$, higher $\mathrm{A} \beta 42$ concentrations differentiated iNPH with a sensitivity of 0.813 (95\% CI $0.636-$ 0.928 ) and a specificity of 0.506 (95\% CI $0.393-0.619$ ). The PLR and NLR of CSF A $\beta 42$ concentrations in differentiating iNPH from AD were 2.032 (95\% CI $0.918-$ 4.498) and 0.324 (95\% CI 0.156-0.673), respectively.

Relative to AD, the sensitivity and specificity of lower CSF $\mathrm{t}$-tau concentrations in differentiating $\mathrm{iNPH}$ were

Table 3 Summary of the diagnostic results of the included studies

\begin{tabular}{|c|c|c|c|c|c|}
\hline Study & $\begin{array}{l}\text { Group } \\
\text { compared }\end{array}$ & $\begin{array}{l}\text { Cutoff } \\
\text { value }\end{array}$ & Auc & Sensitivity & Specificity \\
\hline \multirow{3}{*}{$\begin{array}{l}\text { Jingami } \\
\text { et al. [23] }\end{array}$} & iNPH vs. AD & & & & \\
\hline & t-tau & 766 & 0.9 & 75 & 98 \\
\hline & p-tau & 24.4 & 0.91 & 95 & 74 \\
\hline \multirow{4}{*}{$\begin{array}{l}\text { Kapaki et al. } \\
\text { [13] }\end{array}$} & iNPH vs. AD & & & & \\
\hline & $A \beta 42$ & 268 & 0.58 & 90.9 & 44.4 \\
\hline & t-tau & 294 & 0.84 & 92.5 & 77.8 \\
\hline & p-tau & 47.4 & 0.83 & 88.7 & 86.7 \\
\hline \multirow{4}{*}{$\begin{array}{l}\text { Schirinzi } \\
\text { et al. [28] }\end{array}$} & iNPH vs. AD & & & & \\
\hline & $A \beta 42$ & 371 & 0.75 & 73.3 & 81.3 \\
\hline & t-tau & 386 & 0.99 & 100 & 93.8 \\
\hline & p-tau & 46 & 0.99 & 100 & 93.8 \\
\hline
\end{tabular}


0.828 (95\% CI $0.732-0.900)$ and 0.842 (95\% CI $0.756-$ 0.907), respectively. The PLR and NLR of CSF t-tau concentrations in differentiating iNPH were 8.199 (95\% CI $1.738-38.678$ ) and 0.112 (95\% CI $0.018-0.699)$, respectively. The SROC AUC value was $0.963 \pm 0.021$, and the pooled diagnostic accuracy $\left(Q^{*}\right)$ was $0.909 \pm 0.032$.

Compared with $\mathrm{AD}$, the sensitivity and specificity of lower CSF p-tau concentrations in distinguishing iNPH were 0.943 (95\% CI $0.871-0.981)$ and 0.851 (95\% CI 0.767-0.914), respectively. The PLR and NLR of CSF p-tau concentrations in distinguishing iNPH were 5.577 (95\% CI 3.513-8.854) and 0.085 (95\% CI $0.038-0.193)$, respectively. The SROC AUC value was $0.9453 \pm 0.037$, and the pooled diagnostic accuracy $\left(Q^{*}\right)$ was $0.884 \pm 0.048$.

\section{Discussion}

In this systematic review and meta-analysis, we explored whether concentrations of CSF A $\beta 42$, $t$-tau, and p-tau are of potential value in differentiating iNPH from $A D$ and from healthy normal controls. Our results suggest that concentrations of CSF $\mathrm{t}$-tau and p-tau in $\mathrm{NPH}$ patients are lower than in $\mathrm{AD}$ patients and lower than healthy controls. Concentrations of $A \beta 42$ in iNPH patients are lower than in healthy controls but slightly higher than in AD patients. Lower CSF t-tau and p-tau levels appear to carry higher sensitivity and specificity in differentiating $\mathrm{iNPH}$ from $\mathrm{AD}$.

The combined pattern of reduced CSF levels of $A \beta 42$ and increased levels of CSF t-tau and p-tau is an established CSF biomarker for AD [9]. The low A $\beta 42$ levels compared to healthy controls in $\mathrm{AD}$ are believed to result from sequestration of soluble beta amyloid in plaques, while the elevated concentrations of $\mathrm{t}$-tau and $\mathrm{p}$-tau are thought to reflect release from the intraneuronal compartment owing to nerve cell and neurite damage. Past studies have fairly consistently found iNPH patients have low CSF $A \beta 42$ levels in a range that overlaps that of $\mathrm{AD}$. In this meta-analysis, we found that high $\mathrm{A} \beta 42$ levels in CSF might be slightly helpful in differentiating iNPH from $A D$, whereas low CSF $A \beta 42$ could potentially be useful as a marker for differentiating of iNPH from healthy normal elderly.

Various hypotheses have been proposed to explain the reduction of CSF A $\beta 42$ levels in iNPH patients. One hypothesis is that delivery of A $\beta 42$ to the CSF compartment is impaired as a consequence of reduced centripetal flow of extracellular fluid in the brain caused by the retrograde CSF flow dynamics in iNPH $[14,25]$. Xie et al. [29] found that $A \beta$ clearance from the extracellular fluid is increased during sleep, as the interstitial space increases $60 \%$ in size at this time. Hence, Graff-Radford et al. [14] hypothesized that reduced CSF A 342 levels in iNPH may be related to the smaller extracellular space and having less room for the convective flux of CSF and interstitial fluid during sleep. The increased CSF levels of many proteins obtained from lumbar CSF drainage in iNPH provides indirect support for this theory. However, Hladky and Barrand [30] raised doubts about this theory and proposed a hypothesis that the elimination of Abeta from the brain might be associated with the perivascular lymphatic drainage pathways. Another possible explanation is that hypometabolism in the periventricular zone, as sometime seen on PET and SPECT studies in iNPH patients, may play a role in lowering generation of CSF A 342 [25].

Contrary to the consistency of finding low A $\beta 42$ level in $\mathrm{iNPH}$, there is only moderate agreement regarding the concentrations of CSF t-tau and p-tau. In 2007, Kapaki et al. [13] found that t-tau was slightly increased in iNPH and obviously increased in AD compared to healthy controls, while p-tau levels were significantly increased only in AD. Therefore, the authors concluded that CSF p-tau alone or in combination with t-tau may be a useful marker in the differentiation of iNPH from AD. However, other studies found that both $t$-tau and p-tau concentrations were significantly reduced in iNPH patients compared to $\mathrm{AD}$ [23] and healthy controls [25]. Our results suggest that concentrations of CSF $t$-tau and p-tau in iNPH are lower than in AD and healthy controls and this difference might be used to differentiate iNPH from $\mathrm{AD}$ or healthy controls. Prior reports have found that the concentrations of $\mathrm{t}$-tau and $\mathrm{p}$-tau increased with age $[31,32]$. Since the average ages of iNPH patients were older than the $\mathrm{AD}$ patients in this study, one might have expected to find higher $\mathrm{t}$-tau and $\mathrm{p}$-tau levels in the iNPH group. On the contrary, we found the opposite. Hence, the differences in the concentrations of $\mathrm{t}$-tau and $\mathrm{p}$-tau observed between iNPH and AD are more likely associated with the pathophysiology of iNPH than age differences [31]. Reduced clearance from extracellular fluid and decreased brain metabolism of periventricular zone in iNPH may contribute to this phenomenon of reduction in CSF t-tau and p-tau levels in iNPH [14].

Prior studies reported that the ventricular CSF t-tau and p-tau levels are higher than in lumbar CSF samples [26]. In the studies we analyzed, there was marked heterogeneity in CSF levels depending on the location from which the CSF was collected. Therefore we performed subgroup analysis taking into account the CSF sources (lumbar CSF vs. ventricular CSF). Results showed that the lumbar CSF $\mathrm{t}$-tau and $\mathrm{p}$-tau levels in iNPH were lower than in $\mathrm{AD}$, while no differences were found in ventricular CSF samples. This finding may support the hypothesis that lower CSF levels of proteins in iNPH relate to the disturbed circulation of CSF to the lumbar region in this disorder. Other possible explanations for this discrepancy 
include insufficient sample size or methodologic differences such as neuronal injury associated with placing a ventricular catheter which may elevate ventricular CSF tau and p-tau levels [12]. A subgroup analysis was also performed according to the categories of country (Asia vs. Caucasian). Results showed that the CSF A $\beta 42$ and $\mathrm{t}$-tau levels were distinct in different races, which suggested that outcomes may have been influenced by ethnicity. However, insufficient sample size or methodologic differences may also explain this contrast.

Our systematic review and meta-analysis has some limitations that should be acknowledged. First, only a limited number of studies were eligible for inclusion, particularly in the diagnostic analyses, which reduced the power of our meta-analysis. Secondly, we observed marked heterogeneity suggesting that there was a significant difference among the included studies, which can be attributed to variation in sample size, age, ethnicity, CSF source, different metrics (means and medians), and methodologic differences in sample collection and the assays employed. Although random-effects and subgroup analyses were performed, these parameters could not completely explain the heterogeneity. Other factors can be identified that may have contributed to the heterogeneity across studies. We were unable to assess what stage of iNPH (mild, moderate, severe) was included in the respective studies, and this could introduce some of the variance in the CSF results. In addition, we assume that the reported cases were pure iNPH or pure AD, but it is known that these conditions can occur together in many cases. The possible coincident occurrence of iNPH with $\mathrm{AD}$ or complications due to other diseases, could not be evaluated because these factors were not reported in the included articles. This is likely to be an important contributing factor in the heterogeneity $[15,27,33]$. Thirdly, sensitivity analysis of $A \beta 42$ levels between $\mathrm{iNPH}$ and $\mathrm{AD}$, $\mathrm{t}$-tau levels between iNPH and healthy controls indicated that the meta-analysis for these variables had poor reliability. Fourthly, we did not include unpublished and nonpeer reviewed studies and our analysis was restricted to publications in English and Chinese, creating the possibility geographic source biases. Finally, a weak publication bias was found in relation to reports of CSF t-tau levels between iNPH and AD, and p-tau levels between $\mathrm{iNPH}$ and AD. However, there was no significant change in the results after using the Trim and Fill method, which suggests that the influence of publication bias on stability of results was weak.

\section{Conclusion}

Our study suggests that reduced CSF t-tau and p-tau maybe come useful markers for the differentiation of $\mathrm{iNPH}$ from $\mathrm{AD}$ or healthy controls. In addition, low
A 342 levels contribute to distinguish iNPH and AD from healthy controls while $A \beta 42$ levels are statistically slightly higher in iNPH compared to AD. Given the relative paucity of studies included and other limitations in this study, it is reasonable to assume our results should be interpreted with caution. In future, well-designed, largescale prospective studies with well-controlled, standardization of experimental protocols for CSF biomarker measurements are warranted as a step towards improving the diagnosis and differential diagnosis of iNPH in clinical practice.

\section{Additional file}

Additional file 1. Supplement characteristics of studies included in the meta-analysis.

\section{Authors' contributions}

Conceived and designed the experiments: ZYC NR YX YFL. Analyzed the data: ZYC CYL. Contributed reagents/materials/analysis tools: JZ. Wrote the paper: ZYC NR YX. All authors read and approved the final manuscript.

\section{Author details \\ ${ }^{1}$ Department of Neurology, Aviation General Hospital of China Medical University \& Beijing Institute of Translational Medicine, Chinese Academy of Sciences, No. 3 Anwai Beiyuan Road, Chaoyang District, Beijing 100012, China. ${ }^{2}$ Department of Neurology and Neuroscience, Weill Medical College of Cornell University, Cornell Memory Disorders Program, 428 East 72 Street, Suite 500, New York, NY 10021, USA. ${ }^{3}$ Department of Neurology, Peking Union Medical College Hospital, Beijing, China.}

\section{Acknowledgements \\ None.}

Competing interests

The authors declare that they have no competing interests.

Availability of data and materials

All data are included in the manuscript.

\section{Publisher's Note}

Springer Nature remains neutral with regard to jurisdictional claims in published maps and institutional affiliations.

Received: 16 February 2017 Accepted: 28 April 2017

Published online: 10 May 2017

References

1. Adams RD, Fisher CM, Hakim S, Ojemann RG, Sweet WH. Symptomatic occult hydrocephalus with "normal" cerebrospinal-fluid pressure. A treatable syndrome. N Engl J Med. 1965;273:117-26.

2. Akiguchi I, Shirakashi Y, Budka H, Watanabe Y, Watanabe T, Shiino A, et al. Disproportionate subarachnoid space hydrocephalus-outcome and perivascular space. Ann Clin Transl Neurol. 2014;1:562-9.

3. Jaraj D, Rabiei K, Marlow T, Jensen C, Skoog I, Wikkelso C. Prevalence of idiopathic normal-pressure hydrocephalus. Neurology. 2014;82:1449-54.

4. Tanaka N, Yamaguchi S, Ishikawa H, Ishii H, Meguro K. Prevalence of possible idiopathic normal-pressure hydrocephalus in Japan: the Osaki-Tajiri project. Neuroepidemiology. 2009;32:171-5.

5. Martin-Laez R, Caballero-Arzapalo H, Lopez-Menendez LA, ArangoLasprilla JC, Vazquez-Barquero A. Epidemiology of idiopathic normal 
pressure hydrocephalus: a systematic review of the literature. World Neurosurg. 2015;84:2002-9.

6. Brean A, Eide PK. Prevalence of probable idiopathic normal pressure hydrocephalus in a Norwegian population. Acta Neurol Scand. 2008;1 18:48-53.

7. Marmarou A, Young HF, Aygok GA. Estimated incidence of normal pressure hydrocephalus and shunt outcome in patients residing in assistedliving and extended-care facilities. Neurosurg Focus. 2007;22:E1.

8. Klinge P, Hellstrom P, Tans J, Wikkelso C. One-year outcome in the European multicentre study on iNPH. Acta Neurol Scand. 2012;126:145-53.

9. Olsson B, Lautner R, Andreasson U, Ohrfelt A, Portelius E, Bjerke M, et al. CSF and blood biomarkers for the diagnosis of Alzheimer's disease: a systematic review and meta-analysis. Lancet Neurol. 2016;15:673-84.

10. Blennow K, de Leon MJ, Zetterberg H. Alzheimer's disease. Lancet. 2006;368:387-403.

11. Trojanowski JQ, Schuck T, Schmidt ML, Lee VM. Distribution of tau proteins in the normal human central and peripheral nervous system. J Histochem Cytochem. 1989:37:209-15.

12. Agren-Wilsson A, Lekman A, Sjoberg W, Rosengren L, Blennow K, Bergenheim AT, et al. CSF biomarkers in the evaluation of idiopathic normal pressure hydrocephalus. Acta Neurol Scand. 2007;116:333-9.

13. Kapaki EN, Paraskevas GP, Tzerakis NG, Sfagos C, Seretis A, Kararizou E, et al. Cerebrospinal fluid tau, phospho-tau181 and beta-amyloid1-42 in idiopathic normal pressure hydrocephalus: a discrimination from Alzheimer's disease. Eur J Neurol. 2007;14:168-73.

14. Graff-Radford NR. Alzheimer CSF biomarkers may be misleading in normal-pressure hydrocephalus. Neurology. 2014;83:1573-5.

15. Ray B, Reyes PF, Lahiri DK. Biochemical studies in normal pressure hydrocephalus (NPH) patients: change in CSF levels of amyloid precursor protein (APP), amyloid-beta (Aß) peptide and phospho-tau. J Psychiatr Res. 2011;45:539-47.

16. Liberati A, Altman DG, Tetzlaff J, Mulrow C, Gotzsche PC, loannidis JP, Clarke M, et al. The PRISMA statement for reporting systematic reviews and meta-analyses of studies that evaluate healthcare interventions: explanation and elaboration. PLoS Med. 2009;6:e1000100.

17. Wan X, Wang W, Liu J, Tong T. Estimating the sample mean and standard deviation from the sample size, median, range and/or interquartile range. BMC Med Res Methodol. 2014;14:135.

18. Higgins J, Green S. Cochrane handbook for systematic reviews of interventions, Version 5.1.0 [updated March 2011], The Cochrane Collaboration; 2011.

19. Colditz GA, Burdick E, Mosteller F. Heterogeneity in meta-analysis of data from epidemiologic studies: a commentary. Am J Epidemiol. 1995:142:371-82.

20. Egger M, Davey Smith G, Schneider M, Minder C. Bias in meta-analysis detected by a simple, graphical test. BMJ. 1997:315:629-34.
21. Pyykko OT, Lumela M, Rummukainen J, Nerg O, Seppala TT, Herukka SK, et al. Cerebrospinal fluid biomarker and brain biopsy findings in idiopathic normal pressure hydrocephalus. PLoS ONE. 2014;9:e91974.

22. Tsai A, Malek-Ahmadi M, Kahlon V, Sabbagh MN. Differences in cerebrospinal fluid biomarkers between clinically diagnosed idiopathic normal pressure hydrocephalus and Alzheimer's disease. J Alzheimers Dis Parkinsonism. 2014;4(4). doi: 10.4172/2161-0460.1000150.

23. Jingami N, Asada-Utsugi M, Uemura K, Noto R, Takahashi M, Ozaki A, et al. Idiopathic normal pressure hydrocephalus has a different cerebrospinal fluid biomarker profile from Alzheimer's disease. J Alzheimers Dis. 2015:45:109-15.

24. Miyajima M, Nakajima M, Ogino I, Miyata H, Motoi Y, Arai H. Soluble amyloid precursor protein a in the cerebrospinal fluid as a diagnostic and prognostic biomarker for idiopathic normal pressure hydrocephalus. Eur J Neurol. 2013;20:236-42.

25. Jeppsson A, Zetterberg H, Blennow K, Wikkelso C. Idiopathic normal-pressure hydrocephalus: pathophysiology and diagnosis by CSF biomarkers. Neurology. 2013;80:1385-92.

26. Seppala TT, Nerg O, Koivisto AM, Rummukainen J, Puli L, Zetterberg H, et al. CSF biomarkers for Alzheimer disease correlate with cortical brain biopsy findings. Neurology. 2012;78:1568-75.

27. Lim TS, Choi JY, Park SA, Youn YC, Lee HY, Kim BG, et al. Evaluation of coexistence of Alzheimer's disease in idiopathic normal pressure hydrocephalus using ELISA analyses for CSF biomarkers. BMC Neurol. 2014;14:66.

28. Schirinzi T, Sancesario GM, Ialongo C, Imbriani P, Madeo G, Toniolo S, et al. A clinical and biochemical analysis in the differential diagnosis of idiopathic normal pressure hydrocephalus. Front Neurol. 2015;6:86.

29. Xie L, Kang H, Xu Q, Chen MJ, Liao Y, Thiyagarajan M, et al. Sleep drives metabolite clearance from the adult brain. Science. 2013:342:373-7.

30. Hladky SB, Barrand MA. Mechanisms of fluid movement into, through and out of the brain: evaluation of the evidence. Fluids Barriers CNS. 2014;11:26

31. Paternico D, Galluzzi S, Drago V, Bocchio-Chiavetto L, Zanardini R, Pedrini $\mathrm{L}$, et al. Cerebrospinal fluid markers for Alzheimer's disease in a cognitively healthy cohort of young and old adults. Alzheimers Dement. 2012;8:520-7.

32. Sjogren $\mathrm{M}$, Vanderstichele $\mathrm{H}$, Agren $\mathrm{H}$, Zachrisson $\mathrm{O}$, Edsbagge $\mathrm{M}$, Wikkelso $C$, et al. Tau and A 42 in cerebrospinal fluid from healthy adults 21-93 years of age: establishment of reference values. Clin Chem. 2001:47:1776-81.

33. Kudo T, Mima T, Hashimoto R, Nakao K, Morihara T, Tanimukai H, et al. Tau protein is a potential biological marker for normal pressure hydrocephalus. Psychiatry Clin Neurosci. 2000;54:199-202.

\section{Submit your next manuscript to BioMed Central and we will help you at every step:}

- We accept pre-submission inquiries

- Our selector tool helps you to find the most relevant journal

- We provide round the clock customer support

- Convenient online submission

- Thorough peer review

- Inclusion in PubMed and all major indexing services

- Maximum visibility for your research

Submit your manuscript at www.biomedcentral.com/submit
BioMed Central 